\title{
How to Select the Items for the Shopping List of Future High Resolution Electron Microscopists?
}

\author{
D. Van Dyck, ${ }^{*}$ S. Van Aert, ** A.J. den Dekker, ** and A. van den Bos** \\ * University of Antwerp, Department of Physics, Antwerp, 2020, Belgium \\ **Delft University of Technology, Department of Applied Physics, Delft, 2628 CJ, The Netherlands
}

Instrumental developments continue to push the resolution of electron microscopes beyond $1 \AA$. Apart from the continuous improvement in resolution of the classical high resolution electron microscopes (HREM), new possibilities emerge, such as, correction of the spherical aberration both in transmission and scanning transmission electron microscopy (TEM and STEM, respectively), combination of TEM with high angle annular dark field (HAADF) STEM, monochromators, and offaxis holography. Furthermore, in our view, the new electron microscope will show a large versatility in experimental settings under computer control, such as, TEM or STEM, imaging or diffraction techniques (such as convergent beam electron diffraction, precession, and ptychography), focus, voltage, spherical aberration, beam tilt, and crystal tilt. The main limiting factors in the experiment will be the incident electron dose, that is, the amount of electrons that interact with the object during the experiment, and the recording time, because of the radiation sensitivity of the object and the specimen drift, respectively. The question then arises which instrument and which experimental settings are optimal given the incident electron dose or the recording time available. To answer this question, an optimality criterion that meets the purpose of future HREM is needed.

The last decades are characterized by an evolution from macro- to micro- and more recently to nanotechnology. In the future, it will even become possible to compose nanostructures atom by atom. Most of the interesting properties of materials are mainly related to their nanostructure. In parallel, there is an evolution in solid state theory where materials properties are increasingly better understood from first principles theoretical calculations. The merging of these fields will enable materials science to evolve into materials design, that is, from describing and understanding towards predicting of materials properties. If this evolution is to be continued, it is imperative that characterization techniques keep pace. In order to correlate real properties with simulations, atom positions in aperiodic structures should be determined with a precision of the order of $0.01 \AA$.

In principle, quantitative HREM is the most appropriate technique to provide the required precision. The reason for this is that from all possible imaging particles, electrons interact most strongly with matter, hereby providing most information for a given amount of radiation damage [1]. Furthermore, electrons make observations in real space possible. This is an asset for the study of aperiodic structures. Extraction of structure parameters from the images can be formulated as a parameter estimation problem. A parametric model describing the expectations of the intensity observations is needed. The model includes the electron object interaction, the transfer in the microscope, and the image detection. The unknown structure parameters are estimated by fitting the model to the experimentally obtained images using a criterion of goodness of fit. Then, the precision of the estimates of the parameters is limited by the presence of noise.

Following the lines of thought mentioned above, the obvious optimality criterion to be used is the precision of the estimates of the atom positions. Statistical parameter estimation theory allows the 
derivation of an expression for the highest precision of the parameter estimates [2]. Thus, it is possible to compute the lower bound on the standard deviation of the position of an atom. This lower bound, which is called the Cramér Rao Lower Bound (CRLB), is a function of both object and microscope parameters. The availability of the CRLB allows quantitative evaluation, comparison, and optimization of different experimental settings. This process, called statistical experimental design, can be illustrated as follows. Suppose that the microscope is able to visualize an isolated atom and that $\sigma$ is the width of the image of the atom, that is, the Rayleigh resolution. The thus defined resolution will depend on object and microscope parameters only. The CRLB on the standard deviation of the position of the atom, on the other hand, may be shown to be of the order of $\sigma / \sqrt{\mathrm{N}}$, with $\mathrm{N}$ the total number of detected electrons. It is now clear that it is not only the Rayleigh resolution that matters but the electron dose as well.

Recently, progress has been made in the derivation of the optimal probe and detector configuration in ADF STEM [3]. From the evaluations of the CRLB, it follows that the optimal probe is not the narrowest probe possible. Furthermore, it has been found that the radius of the hole in the detector should be as large as the optimal aperture radius. Moreover, by the same token, a monochromator usually doesn't pay off in terms of precision [4]. For example, Fig. 1 shows the CRLB on the standard deviation of the position of a gold [100] atom column as a function of the electron energy spread. Two particular cases have been distinguished. Either the recording time or the incident electron dose has been kept constant, presuming that specimen drift or radiation damage puts a practical limit on the experiment, respectively. Currently, STEM and HRTEM are compared along the same line in order to find out which method is to be preferred.

\section{References}

[1] R. Henderson, Quarterly Reviews of Biophysics 28 (1995) 171.

[2] A. van den Bos and A.J. den Dekker, Advances in Imaging and Electron Physics 117 (2001) 241.

[3] S. Van Aert et al., Ultramicroscopy 90 (2002) 273.

[4] A.J. den Dekker et al., Ultramicroscopy 89 (2001) 275.

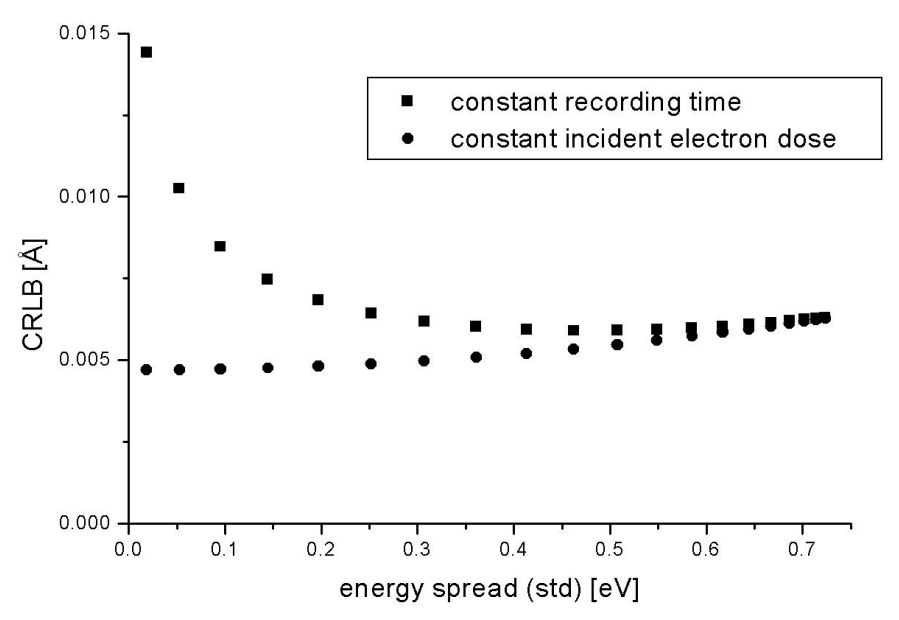

FIG. 1. Lower bound on the standard deviation of the position of a gold [100] atom column as a function of the electron energy spread associated with a constant recording time and a constant incident electron dose, respectively. 